\title{
Magnetohydrodynamic waves in solar partially ionized plasmas: two-fluid approach
}

\author{
T. V. Zaqarashvili ${ }^{1,2}$, M. L. Khodachenko ${ }^{1}$, and H. O. Rucker ${ }^{1}$ \\ 1 Space Research Institute, Austrian Academy of Sciences, Schmiedlstrasse 6, 8042 Graz, Austria \\ e-mail: [teimuraz.zaqarashvili; maxim.khodachenko; rucker] @oeaw.ac.at \\ 2 Abastumani Astrophysical Observatory at Ilia State University, Kazbegi ave. 2a, Tbilisi, Georgia
}

Received 15 December 2010 / Accepted 5 February 2011

\begin{abstract}
Context. Partially ionized plasma is usually described by a single-fluid approach, where the ion-neutral collision effects are expressed by Cowling conductivity in the induction equation. However, the single-fluid approach is not valid for time-scales less than ion-neutral collision time. For these time-scales the two-fluid description is the better approximation.

Aims. We aim to derive the dynamics of magnetohydrodynamic (MHD) waves in two-fluid partially ionized plasmas and to compare the results with those obtained under single-fluid description.

Methods. Two-fluid equations are used, where ion-electron plasma and neutral particles are considered as separate fluids. Dispersion relations of linear waves are derived for the simplest case of homogeneous medium. Frequencies and damping rates of waves are obtained for different parameters of background plasma.

Results. We found that two- and single-fluid descriptions give similar results for low-frequency waves. However, the dynamics of MHD waves in the two-fluid approach is significantly changed when the wave frequency becomes comparable with or higher than the ion-neutral collision frequency. Alfvén and fast magneto-acoustic waves attain their maximum damping rate at particular frequencies (for example, the peak frequency equals 2.5 times the ion-neutral collision frequency for $50 \%$ of neutral hydrogen) in the wave spectrum. The damping rates are reduced for the higher frequency waves. The new mode of slow magneto-acoustic wave appears for higher frequency branch, which is connected to neutral hydrogen fluid.

Conclusions. The single-fluid approach perfectly deals with slow processes in partially ionized plasmas, but fails for time-scales shorter than ion-neutral collision time. Therefore, the two-fluid approximation should be used for the description of relatively fast processes. Some results of the single-fluid description should be revised in future such as the damping of high-frequency Alfvén waves in the solar chromosphere due to ion-neutral collisions.
\end{abstract}

Key words. Sun: atmosphere - Sun: oscillations

\section{Introduction}

Astrophysical plasmas often are partially ionized. Neutral atoms may change the plasma dynamics through collisions with charged particles. The ion-neutral collisions may lead to different new phenomena in the plasma, for example the damping of magnetohydrodynamic (MHD) waves (Khodachenko et al. 2004; Forteza et al. 2007). The solar photosphere, the chromosphere, and the prominences contain a significant amount of neutral atoms, therefore the complete description of plasma processes requires the consideration of partial ionization effects.

Braginskii (1965) gave the basic principles of transport processes in plasma including the effects of partial ionization. Since this review, numerous papers addressed the problem of partial ionization in the different regions of solar atmosphere. Khodachenko \& Zaitsev (2002) studied the formation of the magnetic flux tube in a converging flow of the solar photosphere, while Vranjes et al. (2008) studied the Alfvén waves in weakly ionized photospheric plasma. Leake \& Arber (2005) and Arber et al. (2007) studied the effect of partially ionized plasma on emerging magnetic flux tubes and concluded that the chromospheric neutrals may transform the magnetic tube into forcefree configuration. Haerendel (1992), De Pontieu \& Haerendel (1998), James \& Erdélyi (2002), and James et al. (2004) considered the damping of Alfvén waves through ion-neutral collision as a mechanism of spicule formation. Khodachenko el al. (2004) and Leake et al. (2006) studied the importance of ion-neutral collisions in the damping of MHD waves in the chromosphere and prominences. Forteza et al. (2007, 2008), Soler et al. (2009a, 2009b, 2010), and Carbonell et al. (2010) studied the damping of MHD waves in partially ionized prominence plasma with and without plasma flow.

All these papers considered the single-fluid MHD approach when the inertial terms in the momentum equation of the relative velocity between ions and neutrals are neglected. The partially ionized plasma effects are described by a generalized Ohm's law with Cowling conductivity, which leads to the modified induction equation (Khodachenko el al. 2004). Ambipolar diffusion is more pronounced during the transverse motion of plasma with regard to the magnetic field, therefore the Alfvén and fast magneto-acoustic waves are more efficiently damped. The slow magneto-acoustic waves are weakly damped in the low plasma beta case. Moreover, Forteza et al. (2007) found that the damping rate of slow magneto-acoustic waves derived through a normal mode analysis is different from that estimated by Braginskii (1965). The cause of the discrepancy between the normal mode analysis (Forteza et al. 2007) and the energy consideration (Braginskii 1965) is still an open question, and the present study attempts to shed light on it. 
The single-fluid approach has been shown to be valid for the time-scales that are longer than the ion-neutral collision time. However, the approximation fails for the shorter time-scales, therefore the two-fluid approximation, which means the treatment of ion-electron and neutral gases as separate fluids, should be considered. The two-fluid approximation is valid for timescales longer than the ion-electron collision time, which is significantly shorter because of the Coulomb collision between ions and electrons.

In this paper, we study MHD waves in two-fluid partially ionized plasma. We pay particular attention to the wave damping through ion-neutral collisions and compare the wave dynamics in single and two-fluid approximations. We derive the twofluid MHD equations from initial three-fluid equations and solve the linearized equations in the simplest case of a homogeneous plasma.

\section{Main equations}

We aim to study partially ionized plasma, which consists of electrons, ions, and neutral atoms. We assume that each species has a Maxwell velocity distribution, therefore they can be described as separate fluids. Below we first write the equations in threefluid description and then perform the consequent transition to two-fluid and single-fluid approaches.

\subsection{Three-fluid equations}

The fluid equations for each species can be derived from Boltzmann kinetic equations, which have the forms (Braginskii 1965; Goedbloed \& Poedts 2004)

$$
\begin{aligned}
& \frac{\partial n_{\mathrm{e}}}{\partial t}+\nabla \cdot\left(n_{\mathrm{e}} \boldsymbol{V}_{\mathrm{e}}\right)=0, \\
& \frac{\partial n_{\mathrm{i}}}{\partial t}+\nabla \cdot\left(n_{\mathrm{i}} \boldsymbol{V}_{\mathrm{i}}\right)=0 \\
& \frac{\partial n_{\mathrm{n}}}{\partial t}+\nabla \cdot\left(n_{\mathrm{n}} \boldsymbol{V}_{\mathrm{n}}\right)=0, \\
& m_{\mathrm{e}} n_{\mathrm{e}}\left(\frac{\partial \boldsymbol{V}_{\mathrm{e}}}{\partial t}+\left(\boldsymbol{V}_{\mathrm{e}} \cdot \nabla\right) \boldsymbol{V}_{\mathrm{e}}\right)=-\nabla p_{\mathrm{e}}-\nabla \cdot \pi_{\mathrm{e}} \\
& \quad-e n_{\mathrm{e}}\left(\boldsymbol{E}+\frac{1}{c} \boldsymbol{V}_{\mathrm{e}} \times \boldsymbol{B}\right)+\boldsymbol{R}_{\mathrm{e}}, \\
& m_{\mathrm{i}} n_{\mathrm{i}}\left(\frac{\partial \boldsymbol{V}_{\mathrm{i}}}{\partial t}+\left(\boldsymbol{V}_{\mathrm{i}} \cdot \nabla\right) \boldsymbol{V}_{\mathrm{i}}\right)=-\nabla p_{\mathrm{i}}-\nabla \cdot \pi_{\mathrm{i}} \\
& \quad+Z e n_{\mathrm{i}}\left(\boldsymbol{E}+\frac{1}{c} \boldsymbol{V}_{\mathrm{i}} \times \boldsymbol{B}\right)+\boldsymbol{R}_{\mathrm{i}}, \\
& m_{\mathrm{n}} n_{\mathrm{n}}\left(\frac{\partial \boldsymbol{V}_{\mathrm{n}}}{\partial t}+\left(\boldsymbol{V}_{\mathrm{n}} \cdot \nabla\right) \boldsymbol{V}_{\mathrm{n}}\right)=-\nabla p_{\mathrm{n}}-\nabla \cdot \pi_{\mathrm{n}}+\boldsymbol{R}_{\mathrm{n}}, \\
& \frac{3}{2} n_{\mathrm{e}} k\left(\frac{\partial T_{\mathrm{e}}}{\partial t}+\left(\boldsymbol{V}_{\mathrm{e}} \cdot \nabla\right) T_{\mathrm{e}}\right)+p_{\mathrm{e}} \nabla \cdot \boldsymbol{V}_{\mathrm{e}}+\pi_{\mathrm{e}}: \nabla \boldsymbol{V}_{\mathrm{e}}=-\nabla \cdot \boldsymbol{q}_{\mathrm{e}}+Q_{\mathrm{e}} \\
& \frac{3}{2} n_{\mathrm{i}} k\left(\frac{\partial T_{\mathrm{i}}}{\partial t}+\left(\boldsymbol{V}_{\mathrm{i}} \cdot \nabla\right) T_{\mathrm{i}}\right)+p_{\mathrm{i}} \nabla \cdot \boldsymbol{V}_{\mathrm{i}}+\pi_{\mathrm{i}}: \nabla \boldsymbol{V}_{\mathrm{i}}=-\nabla \cdot \boldsymbol{q}_{\mathrm{i}}+Q_{\mathrm{i}} \\
& \frac{3}{2} n_{\mathrm{n}}\left(\frac{\partial T_{\mathrm{n}}}{\partial t}+\left(\boldsymbol{V}_{\mathrm{n}} \cdot \nabla\right) T_{\mathrm{n}}\right)+p_{\mathrm{n}} \nabla \cdot \boldsymbol{V}_{\mathrm{n}}+\pi_{\mathrm{n}}: \nabla \boldsymbol{V}_{\mathrm{n}}=-\nabla \cdot \boldsymbol{q}_{\mathrm{n}}+Q_{\mathrm{n}} \\
& p_{\mathrm{i}} k T_{\mathrm{i}}, p_{\mathrm{n}}=n_{\mathrm{n}} k T_{\mathrm{n}}, \\
& \hline
\end{aligned}
$$

where $m_{a}, n_{a}, p_{a}, T_{a}, \boldsymbol{V}_{a}$ are the mass, the density, the pressure, the temperature and the velocity of particles $a, \boldsymbol{E}$ is the electric field, $\boldsymbol{B}$ is the magnetic field strength, $\boldsymbol{q}_{a}$ is the heat flux density of particles $a, \boldsymbol{R}_{a}$ is the change of impulse of particles $a$ through collisions with other sort of particles, $Q_{a}$ is the heat production through collisions of particles $a$ with other sort of particles, $\pi_{a}$ is the off-diagonal pressure tensor of particles $a, e=4.8 \times 10^{-10}$ statcoul is the electron charge, $c=2.9979 \times 10^{10} \mathrm{~cm} \mathrm{~s}^{-1}$ is the speed of light and $k=1.38 \times 10^{-16} \mathrm{erg} \mathrm{\textrm {K } ^ { - 1 }}$ is the Boltzmann constant. The double dot indicates that a double sum over the Cartesian components is to be taken. The plasma is supposed to be quasi-neutral, which means $n_{\mathrm{e}}=Z n_{\mathrm{i}}$. Below we consider hydrogen ions and hydrogen neutral atoms that imply $Z=1$. The description of the system is completed by Maxwell equations, which have the forms (without displacement current)

$$
\begin{aligned}
& \nabla \times \boldsymbol{E}=-\frac{1}{c} \frac{\partial \boldsymbol{B}}{\partial t}, \\
& \nabla \times \boldsymbol{B}=\frac{4 \pi}{c} \boldsymbol{j},
\end{aligned}
$$

where

$\boldsymbol{j}=-e n_{\mathrm{e}}\left(\boldsymbol{V}_{\mathrm{e}}-\boldsymbol{V}_{\mathrm{i}}\right)=-e n_{\mathrm{e}} \boldsymbol{u}$

is the current density.

For a Maxwell distribution in each species, $\boldsymbol{R}_{a}$ and $Q_{a}$ are expressed as (Braginskii 1965):

$$
\begin{aligned}
& \boldsymbol{R}_{\mathrm{e}}=-\alpha_{\mathrm{ei}}\left(\boldsymbol{V}_{\mathrm{e}}-\boldsymbol{V}_{\mathrm{i}}\right)-\alpha_{\mathrm{en}}\left(\boldsymbol{V}_{\mathrm{e}}-\boldsymbol{V}_{\mathrm{n}}\right), \\
& \boldsymbol{R}_{\mathrm{i}}=-\alpha_{\mathrm{ie}}\left(\boldsymbol{V}_{\mathrm{i}}-\boldsymbol{V}_{\mathrm{e}}\right)-\alpha_{\mathrm{in}}\left(\boldsymbol{V}_{\mathrm{i}}-\boldsymbol{V}_{\mathrm{n}}\right), \\
& \boldsymbol{R}_{\mathrm{n}}=-\alpha_{\mathrm{ne}}\left(\boldsymbol{V}_{\mathrm{n}}-\boldsymbol{V}_{\mathrm{e}}\right)-\alpha_{\mathrm{ni}}\left(\boldsymbol{V}_{\mathrm{n}}-\boldsymbol{V}_{\mathrm{i}}\right), \\
& Q_{\mathrm{e}}=\alpha_{\mathrm{ei}}\left(\boldsymbol{V}_{\mathrm{e}}-\boldsymbol{V}_{\mathrm{i}}\right) \boldsymbol{V}_{\mathrm{e}}+\alpha_{\mathrm{en}}\left(\boldsymbol{V}_{\mathrm{e}}-\boldsymbol{V}_{\mathrm{n}}\right) \boldsymbol{V}_{\mathrm{e}}, \\
& Q_{\mathrm{i}}=\alpha_{\mathrm{ie}}\left(\boldsymbol{V}_{\mathrm{i}}-\boldsymbol{V}_{\mathrm{e}}\right) \boldsymbol{V}_{\mathrm{i}}+\alpha_{\mathrm{in}}\left(\boldsymbol{V}_{\mathrm{i}}-\boldsymbol{V}_{\mathrm{n}}\right) \boldsymbol{V}_{\mathrm{i}}, \\
& Q_{\mathrm{n}}=\alpha_{\mathrm{ne}}\left(\boldsymbol{V}_{\mathrm{n}}-\boldsymbol{V}_{\mathrm{e}}\right) \boldsymbol{V}_{\mathrm{n}}+\alpha_{\mathrm{ni}}\left(\boldsymbol{V}_{\mathrm{n}}-\boldsymbol{V}_{\mathrm{i}}\right) \boldsymbol{V}_{\mathrm{n}},
\end{aligned}
$$

where $\alpha_{a b}=\alpha_{b a}$ are the coefficients of friction between particles $a$ and $b$.

For time-scales longer than the ion-electron collision time, the electron and ion gases can be considered as a single fluid. This significantly simplifies the equations, taking into account the smallness of electron mass with regard to the masses of ion and neutral atoms. Then the three-fluid description can be changed to the two-fluid description, where one component is the ion-electron gas and the second component is the gas of neutral atoms.

\subsection{Two-fluid equations}

Summing of Eqs. (4) and (5), Eqs. (7) and (8), and first two equations of Eq. (10), we obtain (after neglecting the electron inertia and the viscosity effect expressed by the off-diagonal pressure tensor $\pi_{a}$ )

$$
\frac{\partial n_{\mathrm{i}}}{\partial t}+\nabla \cdot\left(n_{\mathrm{i}} \boldsymbol{V}_{\mathrm{i}}\right)=0
$$




$$
\begin{aligned}
& \frac{\partial n_{\mathrm{n}}}{\partial t}+\nabla \cdot\left(n_{\mathrm{n}} \boldsymbol{V}_{\mathrm{n}}\right)=0, \\
& m_{\mathrm{i}} n_{\mathrm{i}}\left(\frac{\partial \boldsymbol{V}_{\mathrm{i}}}{\partial t}+\left(\boldsymbol{V}_{\mathrm{i}} \cdot \nabla\right) \boldsymbol{V}_{\mathrm{i}}\right)=-\nabla p_{\mathrm{ie}}+\frac{1}{c} \boldsymbol{j} \times \boldsymbol{B}+\frac{\alpha_{\mathrm{en}}}{e n_{\mathrm{e}}} \boldsymbol{j} \\
& \quad-\left(\alpha_{\mathrm{in}}+\alpha_{\mathrm{en}}\right)\left(\boldsymbol{V}_{\mathrm{i}}-\boldsymbol{V}_{\mathrm{n}}\right), \\
& m_{\mathrm{n}} n_{\mathrm{n}}\left(\frac{\partial \boldsymbol{V}_{\mathrm{n}}}{\partial t}+\left(\boldsymbol{V}_{\mathrm{n}} \cdot \nabla\right) \boldsymbol{V}_{\mathrm{n}}\right)=-\nabla p_{\mathrm{n}}-\frac{\alpha_{\mathrm{en}}}{e n_{\mathrm{e}}} \\
& \quad+\left(\alpha_{\mathrm{in}}+\alpha_{\mathrm{en}}\right)\left(\boldsymbol{V}_{\mathrm{i}}-\boldsymbol{V}_{\mathrm{n}}\right), \\
& \frac{\partial p_{\mathrm{ie}}}{\partial t}+\left(\boldsymbol{V}_{\mathrm{i}} \cdot \nabla\right) p_{\mathrm{ie}}+\gamma p_{\mathrm{ie}} \nabla \cdot \boldsymbol{V}_{\mathrm{i}}=(\gamma-1) \frac{\alpha_{\mathrm{ei}}}{e^{2} n_{\mathrm{e}}^{2}} j^{2} \\
& \quad+(\gamma-1) \alpha_{\mathrm{in}}\left(\boldsymbol{V}_{\mathrm{i}}-\boldsymbol{V}_{\mathrm{n}}\right) \cdot \boldsymbol{V}_{\mathrm{i}}+(\gamma-1) \alpha_{\mathrm{en}}\left(\boldsymbol{V}_{\mathrm{e}}-\boldsymbol{V}_{\mathrm{n}}\right) \cdot \boldsymbol{V}_{\mathrm{e}} \\
& \quad+\frac{(\boldsymbol{j} \cdot \nabla) p_{\mathrm{e}}}{e n_{\mathrm{e}}}+\gamma p_{\mathrm{e}} \nabla \cdot \frac{\boldsymbol{j}}{e n_{\mathrm{e}}}-(\gamma-1) \nabla \cdot\left(\boldsymbol{q}_{\mathrm{i}}+\boldsymbol{q}_{\mathrm{e}}\right), \\
& \frac{\partial p_{\mathrm{n}}}{\partial t}+\left(\boldsymbol{V}_{\mathrm{n}} \cdot \nabla\right) p_{\mathrm{n}}+\gamma p_{\mathrm{n}} \nabla \cdot \boldsymbol{V}_{\mathrm{n}}=-(\gamma-1) \alpha_{\mathrm{in}}\left(\boldsymbol{V}_{\mathrm{i}}-\boldsymbol{V}_{\mathrm{n}}\right) \cdot \boldsymbol{V}_{\mathrm{n}} \\
& \quad+(\gamma-1) \alpha_{\mathrm{en}}\left(\boldsymbol{V}_{\mathrm{n}}-\boldsymbol{V}_{\mathrm{e}}\right) \cdot \boldsymbol{V}_{\mathrm{n}}-(\gamma-1) \nabla \cdot \boldsymbol{q}_{\mathrm{n}},
\end{aligned}
$$

where $p_{\mathrm{ie}}=p_{\mathrm{i}}+p_{\mathrm{e}}$ is the pressure of ion-electron gas and $\gamma=$ $C_{p} / C_{v}=5 / 3$ is the ratio of specific heats.

Ohm's law is obtained from the electron equation (Eq. (4)) after neglecting the electron inertia (i.e. the left-hand side terms) and it has the form

$$
\begin{aligned}
\boldsymbol{E}+ & \frac{1}{c} \boldsymbol{V}_{\mathrm{i}} \times \boldsymbol{B}+\frac{1}{e n_{\mathrm{e}}} \nabla p_{\mathrm{e}}=\frac{\alpha_{\mathrm{ei}}+\alpha_{\mathrm{en}}}{e^{2} n_{\mathrm{e}}^{2}} \boldsymbol{j}-\frac{\alpha_{\mathrm{en}}}{e n_{\mathrm{e}}}\left(\boldsymbol{V}_{\mathrm{i}}-\boldsymbol{V}_{\mathrm{n}}\right) \\
& +\frac{1}{c e n_{\mathrm{e}}} \boldsymbol{j} \times \boldsymbol{B} .
\end{aligned}
$$

The Maxwell equation (Eq. (11)) and Ohm's law (Eq. (26)) lead to the induction equation

$$
\begin{aligned}
\frac{\partial \boldsymbol{B}}{\partial t}= & \nabla \times\left(\boldsymbol{V}_{\mathrm{i}} \times \boldsymbol{B}\right)+\nabla \times\left(\frac{c \nabla p_{\mathrm{e}}}{e n_{\mathrm{e}}}\right)-\nabla \times(\eta \nabla \times \boldsymbol{B}) \\
& -\nabla \times\left(\frac{\boldsymbol{j} \times \boldsymbol{B}}{e n_{\mathrm{e}}}\right)+\nabla \times\left(\frac{c \alpha_{\mathrm{en}}\left(\boldsymbol{V}_{\mathrm{i}}-\boldsymbol{V}_{\mathrm{n}}\right)}{e n_{\mathrm{e}}}\right),
\end{aligned}
$$

where

$\eta=\frac{c^{2}}{4 \pi \sigma}=\frac{c^{2}\left(\alpha_{\mathrm{ei}}+\alpha_{\mathrm{en}}\right)}{4 \pi e^{2} n_{\mathrm{e}}^{2}}$

is the coefficient of the magnetic diffusion.

The coefficient of friction between ions and neutrals (if they have the same temperature) is calculated as (Braginskii 1965)

$\alpha_{\text {in }}=n_{\mathrm{i}} n_{\mathrm{n}} m_{\text {in }} \sigma_{\text {in }} \frac{4}{3} \sqrt{\frac{8 k T}{\pi m_{\text {in }}}}$,

where $m_{\mathrm{in}}=m_{\mathrm{i}} m_{\mathrm{n}} /\left(m_{\mathrm{i}}+m_{\mathrm{n}}\right)$ is the reduced mass and $\sigma_{\text {in }}=$ $\pi\left(r_{i}+r_{n}\right)^{2}=4 \pi r_{i}^{2}$ is the collision cross section between ions and neutrals.

The collision frequency between ions and neutrals is then

$$
\begin{aligned}
v_{\mathrm{in}}= & \frac{\alpha_{\mathrm{in}}}{m_{\mathrm{i}} n_{\mathrm{i}}+m_{\mathrm{n}} n_{\mathrm{n}}}=\frac{16 \pi r_{\mathrm{i}}^{2}}{3} \frac{n_{\mathrm{i}} n_{\mathrm{n}} m_{\mathrm{in}}}{m_{\mathrm{i}} n_{\mathrm{i}}+m_{\mathrm{n}} n_{\mathrm{n}}} \sqrt{\frac{8 k T}{\pi m_{\mathrm{in}}}} \\
& =\frac{32 \pi r_{\mathrm{i}}^{2}}{3 \sqrt{\pi}} \frac{n_{\mathrm{i}} n_{\mathrm{n}}}{n_{\mathrm{i}}+n_{\mathrm{n}}} \sqrt{\frac{k T}{m_{\mathrm{i}}}} \approx 5 \times 10^{-12} \frac{n_{\mathrm{i}} n_{\mathrm{n}}}{n_{\mathrm{i}}+n_{\mathrm{n}}} \sqrt{T} \mathrm{~s}^{-1},
\end{aligned}
$$

where the atomic cross section $\pi r_{\mathrm{i}}^{2}=8.7974 \times 10^{-17} \mathrm{~cm}^{2}$ is used and $T$ is normalized by $1 K$. The chromospheric temperature of $10^{4} \mathrm{~K}$ and hydrogen ion and neutral number densities of $2.3 \times$ $10^{10} \mathrm{~cm}^{-3}$ and $1.2 \times 10^{10} \mathrm{~cm}^{-3}$ (Fontenla et al. 1990, model FAL3) give the collision frequency as $4 \mathrm{~s}^{-1}$.
For time-scales longer than ion-neutral collision time $\left(1 / v_{\text {in }}\right)$, the system can be considered as a single fluid (the full equations of single-fluid MHD including neutral hydrogen are presented in Appendix A). However, when the time-scales are near to or shorter than the ion-neutral collision time, the single-fluid description is not valid and the two-fluid equations should be considered. Below, in what follows we study the linear MHD waves in a two-fluid description.

\section{Linear MHD waves}

We consider the simplest case of static and homogeneous plasma with a homogeneous unperturbed magnetic field. Then the linearized two-fluid equations following from Eqs. (20)-(25) and (27) are (neglecting the Hall term and the collision between neutrals and electrons i.e. $\left.\alpha_{\text {en }} \ll \alpha_{\text {in }}\right)$

$$
\begin{aligned}
& \frac{\partial \rho_{\mathrm{i}}^{\prime}}{\partial t}+\rho_{\mathrm{i} 0} \nabla \cdot \boldsymbol{v}_{\mathrm{i}}=0, \\
& \frac{\partial \rho_{\mathrm{n}}^{\prime}}{\partial t}+\rho_{\mathrm{n} 0} \nabla \cdot \boldsymbol{v}_{\mathrm{n}}=0 \\
& \rho_{\mathrm{i} 0} \frac{\partial \boldsymbol{v}_{\mathrm{i}}}{\partial t}=-\nabla p_{\mathrm{ie}}^{\prime}-\frac{1}{4 \pi} \nabla\left(\boldsymbol{B}_{0} \cdot \boldsymbol{b}\right)+\frac{1}{4 \pi}\left(\boldsymbol{B}_{0} \cdot \nabla\right) \boldsymbol{b} \\
& +\frac{\alpha_{\mathrm{en}} c}{4 \pi e n_{\mathrm{e}}} \nabla \times \boldsymbol{b}-\alpha_{\mathrm{in}}\left(\boldsymbol{v}_{\mathrm{i}}-\boldsymbol{v}_{\mathrm{n}}\right) \\
& \rho_{\mathrm{n} 0} \frac{\partial \boldsymbol{v}_{\mathrm{n}}}{\partial t}=-\nabla p_{n}^{\prime}-\frac{\alpha_{\mathrm{en}} c}{4 \pi e n_{\mathrm{e}}} \nabla \times \boldsymbol{b}+\alpha_{\mathrm{in}}\left(\boldsymbol{v}_{\mathrm{i}}-\boldsymbol{v}_{\mathrm{n}}\right), \\
& \frac{\partial \boldsymbol{b}}{\partial t}=\left(\boldsymbol{B}_{0} \cdot \nabla\right) \boldsymbol{v}_{\mathrm{i}}-\boldsymbol{B}_{0} \nabla \cdot \boldsymbol{v}_{\mathrm{i}}+\eta \nabla^{2} \boldsymbol{b}+\frac{c \alpha_{\mathrm{en}}}{e n_{\mathrm{e}}} \nabla \times\left(\boldsymbol{v}_{\mathrm{i}}-\boldsymbol{v}_{\mathrm{n}}\right), \\
& \frac{\partial p_{\mathrm{ie}}^{\prime}}{\partial t}+\gamma p_{\mathrm{ie}} \nabla \cdot \boldsymbol{v}_{\mathrm{i}}=0 \\
& \frac{\partial p_{\mathrm{n}}^{\prime}}{\partial t}+\gamma p_{\mathrm{n}} \nabla \cdot \boldsymbol{v}_{\mathrm{n}}=0 \text {, }
\end{aligned}
$$

where $\rho_{i}^{\prime}\left(\rho_{\mathrm{n}}^{\prime}\right)$ are perturbations of the ion (neutral) density, $\boldsymbol{v}_{\mathrm{i}}$ $\left(\boldsymbol{v}_{\mathrm{n}}\right)$ are the perturbations of ion (neutral) velocity, $p_{\mathrm{ie}}^{\prime}\left(p_{\mathrm{n}}^{\prime}\right)$ are the perturbations of ion-electron (neutral) gas pressure, $\boldsymbol{b}$ is the perturbation of the magnetic field, and $\rho_{\mathrm{i} 0}=m_{\mathrm{i}} n_{\mathrm{i} 0}, \rho_{\mathrm{n} 0}=$ $m_{\mathrm{n}} n_{n 0}, p_{\mathrm{ie}}, p_{\mathrm{n}}, \boldsymbol{B}_{0}$ are their unperturbed values, respectively. Equations (31)-(32) and Eqs. (36)-(37) lead to the expressions

$p_{\mathrm{ie}}^{\prime}=c_{\mathrm{si}}^{2} \rho_{i}^{\prime}, \quad p_{\mathrm{n}}^{\prime}=c_{\mathrm{sn}}^{2} \rho_{\mathrm{n}}^{\prime}$

where $c_{\mathrm{si}}=\sqrt{\gamma p_{\mathrm{ie}} / \rho_{\mathrm{i} 0}}$ and $c_{\mathrm{sn}}=\sqrt{\gamma p_{\mathrm{n}} / \rho_{\mathrm{n} 0}}$ are the sound speeds of ion-electron and neutral gases, respectively.

Below we consider the unperturbed magnetic field, $B_{z}$, directed along the $z$ axis and the wave propagation in $x z$ plane i.e. $\partial / \partial y=0$. Then Eqs. (31)-(37) can be split into Alfvén and magneto-acoustic waves.

\subsection{Alfvén waves}

Let us assume that the Alfvén waves are polarized along $y$ axis. We intend to study the damping of Alfvén waves through the collision between ions and neutrals. Therefore, we neglect the magnetic diffusion for simplicity. Then, Eqs. (31)-(37) give

$$
\begin{aligned}
\frac{\partial v_{\mathrm{i} y}}{\partial t} & =\frac{B_{z}}{4 \pi \rho_{\mathrm{i} 0}} \frac{\partial b_{y}}{\partial z}-\frac{\alpha_{\mathrm{in}}}{\rho_{\mathrm{i} 0}}\left(v_{\mathrm{i} y}-v_{\mathrm{n} y}\right), \\
\frac{\partial v_{\mathrm{n} y}}{\partial t} & =\frac{\alpha_{\mathrm{in}}}{\rho_{\mathrm{n} 0}}\left(v_{\mathrm{i} y}-v_{\mathrm{n} y}\right), \\
\frac{\partial b_{y}}{\partial t} & =B_{z} \frac{\partial v_{\mathrm{i} y}}{\partial z}
\end{aligned}
$$


Fourier analyses assuming disturbances to be proportional to $\exp \left[\mathrm{i}\left(k_{z} z-\omega t\right)\right]$ give the dispersion relation

$a \xi_{\mathrm{i}} \xi_{\mathrm{n}} \varpi^{3}+\mathrm{i} \varpi^{2}-a \xi_{\mathrm{n}} \varpi-\mathrm{i}=0$

where

$\varpi=\frac{\omega}{k_{z} v_{\mathrm{A}}}, a=\frac{k_{z} v_{\mathrm{A}}}{v_{\mathrm{in}}}, \xi_{\mathrm{i}}=\frac{\rho_{\mathrm{i}}}{\rho_{0}}, \quad \xi_{\mathrm{n}}=\frac{\rho_{\mathrm{n}}}{\rho_{0}}, v_{\mathrm{A}}=\frac{B_{z}}{\sqrt{4 \pi \rho_{0}}}$,

$v_{\mathrm{in}}=\frac{\alpha_{\mathrm{in}}}{\rho_{0}}, \rho_{0}=\rho_{\mathrm{i} 0}+\rho_{\mathrm{n} 0}$.

The same dispersion relation can be obtained from linear singlefluid equations by retaining the inertial term in Eq. (A.6). The dispersion relation of the Alfvén waves in linear single-fluid equations without the inertial term can be easily derived as

$\varpi^{2}+\mathrm{i} a \xi_{\mathrm{n}}^{2} \varpi-1=0$.

The solution of Eq. (44) is

$\varpi=\frac{-\mathrm{i} a \xi_{\mathrm{n}}^{2} \pm \sqrt{-a^{2} \xi_{\mathrm{n}}^{4}+4}}{2}$

which for $a \xi_{\mathrm{n}}^{2}<2$ gives the damping rate

$2 \omega_{\mathrm{i}}=\frac{\xi_{\mathrm{n}}^{2} B_{z}^{2}}{4 \pi \alpha_{\mathrm{in}}} k_{z}^{2}$

in full coincidence with Braginskii (1965). In contrast, the condition $a \xi_{\mathrm{n}}^{2}>2$ in Eq. (46) retains only the imaginary part, which gives the cut-off wave number

$k_{\mathrm{c}}=\frac{2 v_{\mathrm{in}}}{\xi_{\mathrm{n}}^{2} v_{\mathrm{A}}}$.

The value of the cut-off wave number has been obtained recently by Barcélo et al. (2010). Hence, the waves with a higher wave number than $k_{\mathrm{c}}$ are evanescent. However, it might be an incomplete conclusion because the complete treatment requires the inclusion of inertial terms, and therefore dealing with Eq. (42) instead of Eq. (44). The first term in Eq. (42) is important for the high-frequency part of the wave spectrum and could not be neglected. We demonstrate it by the solutions of Eqs. (44) and (42).

Figure 1 displays the solutions of the single-fluid (Eq. (44), blue lines) and two-fluid (Eq. (42), red asterisks) dispersion relations for $\xi_{\mathrm{n}}=0.5$. We see that the frequencies and damping rates of Alfvén waves are the same in the single-fluid and twofluid approaches for the low-frequency branch of the spectrum (small $a$ ). But the behavior is dramatically changed when the wave frequency becomes comparable with or higher than the ion-neutral collision frequency, $v_{\text {in }}$, i.e. for $a>1$. The damping time linearly increases with $a$ and the wave frequency becomes zero at some point in the single-fluid case (blue lines). The point where the wave frequency becomes zero corresponds to the cut-off wave number $k_{\mathrm{c}}$ of Barcélo et al. (2010). However, there is no cut-off wave number in solutions of two-fluid dispersion relation (red asterisks): Eq. (42) always has a solution with a real part. Therefore, the occurrence of the cut-off wave number in the single-fluid description is the result of neglecting the inertial terms in the momentum equation of relative velocity between ions and neutrals. Therefore, Eq. (42) is the correct dispersion relation for the whole spectrum of waves. But the dispersion relation (44) is still a good approximation for the lower frequency part of spectrum. Another interesting point of the two-fluid
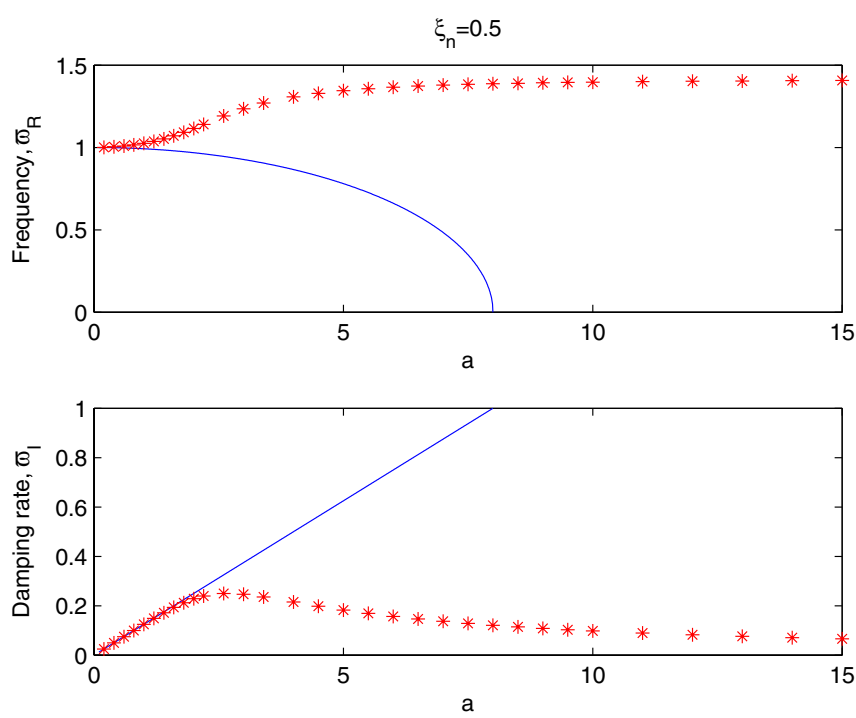

Fig. 1. Alfvén wave frequency vs. normalized wave number. The top (bottom) panel shows the real (imaginary) part of the normalized frequency $\varpi=\omega / k_{z} v_{\mathrm{A}}$ vs. the normalized wavenumber $a=k_{z} v_{\mathrm{A}} / v_{\mathrm{in}}$. The blue line corresponds to the solution of the single-fluid dispersion relation, i.e. Eq. (44) and red asterisks are the solutions of the two-fluid dispersion relation, Eq. (42). The values are calculated for $50 \%$ of neutral hydrogen, $\xi_{\mathrm{n}}=0.5$.

approach is that the damping rate (i.e. $\omega_{I}$ ) attains its maximal value at some wave-lengths for which $a \approx 2.5$. The damping rate decreases for smaller and larger $a$. This means that the waves, which have the frequency in the interval $v_{\text {in }}<\omega<10 v_{\text {in }}$, have stronger damping than other harmonics of the spectrum. This is totally different from the single-fluid solutions, which show the linear increase of damping rate with increasing wave number (lower panel, blue line).

Figure 2 displays the same solutions as in the Fig. 1, but for $\xi_{\mathrm{n}}=0.1$. The solutions have basically the same properties as those with $\xi_{\mathrm{n}}=0.5$. However, the wave length with the maximal damping rate is now shifted to $a \approx 10$.

\subsection{Magneto-acoustic waves}

Now let us turn to magneto-acoustic waves. We consider the waves and wave vectors polarized in $x z$ plane. Then Eqs. (31)(37) are written as (magnetic diffusion is again neglected)

$$
\begin{aligned}
& \frac{\partial \rho_{\mathrm{i}}^{\prime}}{\partial t}+\rho_{\mathrm{i} 0}\left(\frac{\partial v_{\mathrm{i} x}}{\partial x}+\frac{\partial v_{\mathrm{i} z}}{\partial z}\right)=0 \\
& \frac{\partial \rho_{\mathrm{n}}^{\prime}}{\partial t}+\rho_{\mathrm{n} 0}\left(\frac{\partial v_{\mathrm{n} x}}{\partial x}+\frac{\partial v_{\mathrm{n} z}}{\partial z}\right)=0 \\
& \frac{\partial v_{\mathrm{i} x}}{\partial t}=-\frac{1}{\rho_{\mathrm{i} 0}} \frac{\partial p_{\mathrm{ie}}^{\prime}}{\partial x}-\frac{B_{z}}{4 \pi \rho_{\mathrm{i} 0}} \frac{\partial b_{z}}{\partial x}+\frac{B_{z}}{4 \pi \rho_{\mathrm{i} 0}} \frac{\partial b_{x}}{\partial z}-\frac{\alpha_{\mathrm{in}}}{\rho_{\mathrm{i} 0}}\left(v_{\mathrm{i} x}-v_{\mathrm{n} x}\right), \\
& \frac{\partial v_{\mathrm{i} z}}{\partial t}=-\frac{1}{\rho_{\mathrm{i} 0}} \frac{\partial p_{\mathrm{ie}}^{\prime}}{\partial z}-\frac{\alpha_{\mathrm{in}}}{\rho_{\mathrm{i} 0}}\left(v_{\mathrm{i} z}-v_{\mathrm{n} z}\right) \\
& \frac{\partial v_{\mathrm{n} x}}{\partial t}=-\frac{1}{\rho_{\mathrm{n} 0}} \frac{\partial p_{\mathrm{n}}^{\prime}}{\partial x}+\frac{\alpha_{\mathrm{in}}}{\rho_{\mathrm{n} 0}}\left(v_{\mathrm{i} x}-v_{\mathrm{n} x}\right)
\end{aligned}
$$



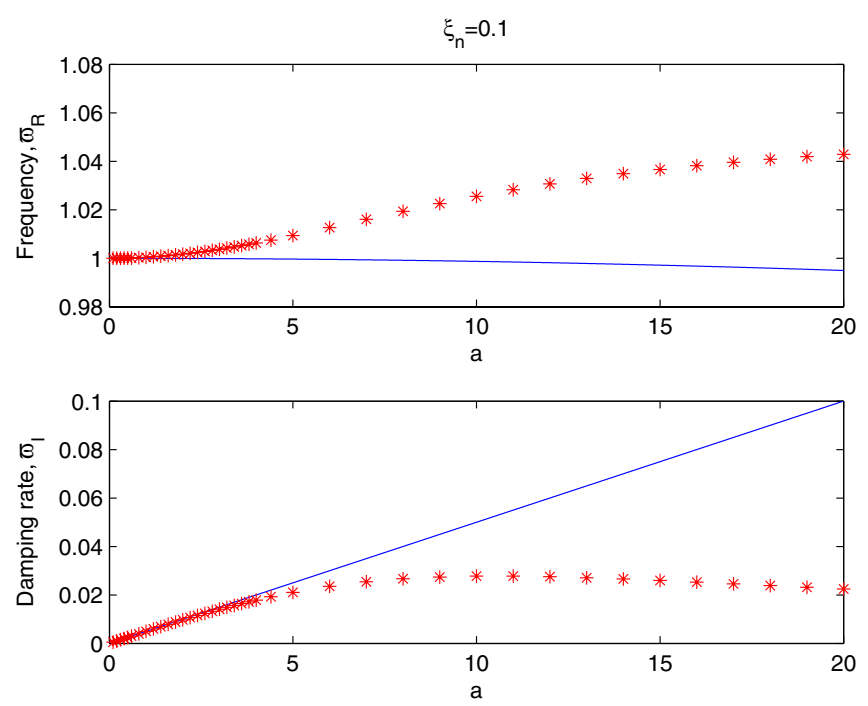

Fig. 2. Same as in Fig. 1, but for $10 \%$ of neutral hydrogen, $\xi_{\mathrm{n}}=0.1$.

$\frac{\partial v_{\mathrm{n} z}}{\partial t}=-\frac{1}{\rho_{\mathrm{n} 0}} \frac{\partial p_{\mathrm{n}}^{\prime}}{\partial z}+\frac{\alpha_{\mathrm{in}}}{\rho_{\mathrm{n} 0}}\left(v_{\mathrm{i} z}-v_{\mathrm{n} z}\right)$,

$\frac{\partial b_{x}}{\partial t}=B_{z} \frac{\partial v_{\mathrm{i} x}}{\partial z}$

$\frac{\partial p_{\mathrm{ie}}^{\prime}}{\partial t}+\gamma p_{\mathrm{ie}}\left(\frac{\partial v_{\mathrm{i} x}}{\partial x}+\frac{\partial v_{\mathrm{i} z}}{\partial z}\right)=0$,

$\frac{\partial p_{\mathrm{n}}^{\prime}}{\partial t}+\gamma p_{\mathrm{n}}\left(\frac{\partial v_{\mathrm{n} x}}{\partial x}+\frac{\partial v_{\mathrm{nz}}}{\partial z}\right)=0$.

A Fourier analysis with $\exp \left[\mathrm{i}\left(k_{x} x+k_{z} z-\omega t\right)\right]$ and some algebra give the dispersion relation

$v_{\text {in }}^{2} \omega\left[\omega^{4}-k^{2}\left(c_{\mathrm{si}}^{2} \xi_{\mathrm{i}}+c_{\mathrm{sn}}^{2} \xi_{\mathrm{n}}+V_{\mathrm{A}}^{2}\right) \omega^{2}+\left(c_{\mathrm{si}}^{2} \xi_{\mathrm{i}}+c_{\mathrm{sn}}^{2} \xi_{\mathrm{n}}\right) k^{2} k_{z}^{2} V_{\mathrm{A}}^{2}\right]$

$-\xi_{\mathrm{i}} \xi_{\mathrm{n}}^{2} \omega\left(\omega^{2}-c_{\mathrm{sn}}^{2} k^{2}\right)\left[\xi_{\mathrm{i}} \omega^{4}-k^{2} V_{\mathrm{A}}^{2} \omega^{2}+c_{\mathrm{si}}^{2} k^{2}\left(k_{z}^{2} V_{\mathrm{A}}^{2}-\xi_{\mathrm{i}} \omega^{2}\right)\right]$

$-\mathrm{i} v_{\mathrm{in}} \xi_{\mathrm{n}}\left[\left(\xi_{\mathrm{n}}-2\right) k^{2} V_{\mathrm{A}}^{2} \omega^{4}+2 \xi_{\mathrm{i}} \omega^{6}+c_{\mathrm{sn}}^{2} k^{2} \omega^{2}\left(k^{2} V_{\mathrm{A}}^{2}+\left(\xi_{\mathrm{n}}^{2}-1\right) \omega^{2}\right)\right.$

$\left.+c_{\mathrm{s}}^{2} \xi_{\mathrm{i}} k^{2}\left(2 k_{z}^{2} V_{\mathrm{A}}^{2} \omega^{2}+\left(\xi_{\mathrm{n}}-2\right) \omega^{4}+c_{\mathrm{sn}}^{2} k^{2}\left(\omega^{2}-k_{z}^{2} V_{\mathrm{A}}^{2}\right)\right)\right]=0$,

where $k=\sqrt{k_{x}^{2}+k_{z}^{2}}$.

The dispersion relation (57) is a seventh order equation with $\omega$, therefore it has seven different solutions. For smaller wave numbers (or lower frequencies) four of the solutions represent the usual magneto-acoustic waves, while three other solutions are purely imaginary and are probably connected to the vortex modes (with $\operatorname{Re}(\omega)=0$ ) that damped through ion neutral collisions. The vortex modes are solutions of fluid equations and they correspond to the fluid vorticity. The vortex modes have zero frequency in the ideal fluid, but may gain a purely imaginary frequency if dissipative processes are evolved. The two vortex modes are transformed into oscillatory modes for shorter wavelengths (see the next paragraph). Then we have two fast magneto-acoustic modes, four slow magneto-acoustic modes, and one vortex solution with a purely imaginary part. Below we consider that the temperatures of all three species are equal i.e. $T_{\mathrm{i}}=T_{\mathrm{e}}=T_{\mathrm{n}}$, which gives $c_{\mathrm{si}}^{2}=\gamma p_{\mathrm{ie}} / \rho_{\mathrm{i} 0}=\gamma\left(p_{\mathrm{i}}+p_{\mathrm{e}}\right) / \rho_{\mathrm{i} 0}=$ $\gamma k\left(T_{\mathrm{i}}+T_{\mathrm{e}}\right) / m_{\mathrm{i}}=2 \gamma k T_{\mathrm{n}} / m_{\mathrm{n}}=2 \gamma p_{\mathrm{n}} / \rho_{\mathrm{n} 0}=2 c_{\mathrm{sn}}^{2}$.

Figure 3 displays all oscillatory solutions of the two-fluid dispersion relation for $\xi_{\mathrm{n}}=0.5$ (only the modes with positive frequencies are shown). The wave propagation is parallel
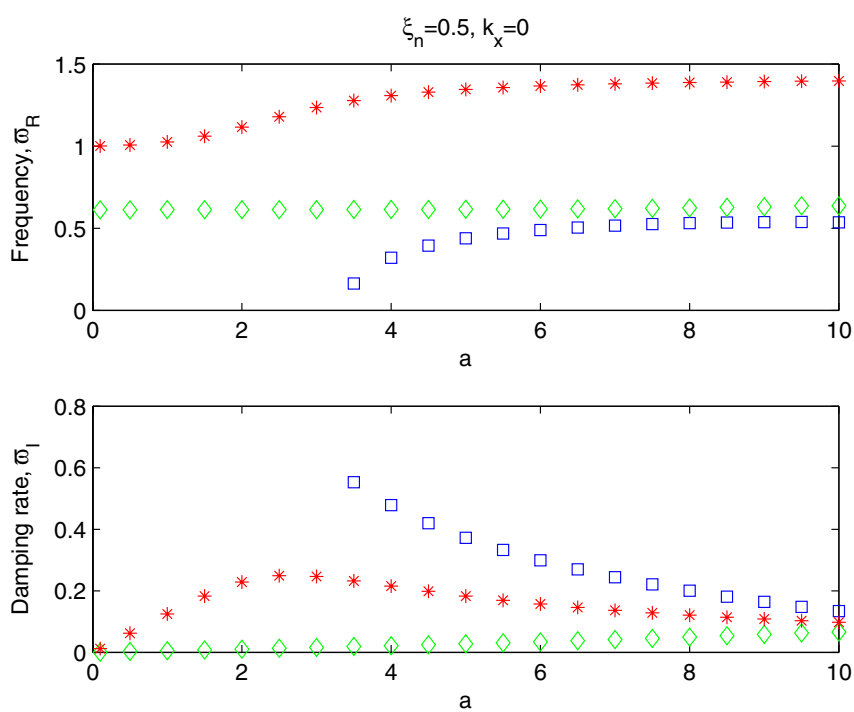

Fig. 3. Frequency and damping rate of different wave modes in twofluid MHD vs. the normalized wavenumber $a=k_{z} v_{\mathrm{A}} / v_{\text {in }}$. Frequencies and damping rates are normalized by $k v_{\mathrm{A}}$. Red asterisks correspond to the fast magneto-acoustic mode and green diamonds correspond to the usual slow magneto-acoustic mode. The mode with the blue squares is the new sort of slow magneto-acoustic wave ("neutral" slow mode), which arises for larger wave numbers. This mode has only an imaginary frequency for small wave numbers, which is not shown in this figure. Frequencies and damping rates are calculated for the waves propagating along the magnetic field. The neutral hydrogen is taken to be $50 \%\left(\xi_{\mathrm{n}}=\right.$ $0.5)$. Here we consider $c_{\mathrm{sn}} / v_{\mathrm{A}}=0.5$.

to the magnetic field and we use $c_{\mathrm{sn}} / v_{\mathrm{A}}=0.5$, where $c_{\mathrm{sn}}$ is the sound speed of neutral hydrogen. For smaller wave numbers, $k<3.5 v_{\text {in }} / v_{\mathrm{A}}$, there are two normal magneto-acoustic modes, fast (red asterisks) and slow (green diamonds). However, for larger wave-numbers, $k>3.5 v_{\text {in }} / v_{\mathrm{A}}$ one additional sort of slow magneto-acoustic mode with a strong damping rate (blue squares) arises. The "neutral" slow mode is connected with neutral atoms. For the higher frequency range, i.e. for a higher than ion-neutral collision frequency, the neutral gas does not feel the ions, therefore it supports the propagation of the additional oscillatory wave mode. This mode obviously disappears for lower frequencies because the collisions couple ions and neutrals and they behave as a single fluid. In other words, for lower frequencies (or small wave numbers) this mode has a zero real part, but a non-zero imaginary part (not shown in the figure). Therefore, the more correct statement is that the oscillatory mode transforms into non-oscillatory vortex mode for smaller wave numbers. The fast magneto-acoustic modes decouple from the slow waves for the parallel propagation and show the same behavior as Alfvén waves. Therefore, the plot of fast magnetoacoustic waves is similar to that of Alfvén waves (see Fig. 1).

It is useful to compare the solutions of two-fluid dispersion relation with those obtained in the single-fluid approach. The damping of fast and slow magneto-acoustic waves has been derived from the energy equation by Braginskii (1965), Khodachenko et al. (2004), Khodachenko \& Rucker (2005), and through a normal mode analysis by Forteza et al. (2007). Damping rates are the same in both considerations for fast magneto-acoustic waves, but they disagree for slow magneto-acoustic waves (Forteza et al. 2007). Namely, slow magneto-acoustic waves show damping for purely parallel propagation in the case of Braginskii, while the damping is absent 

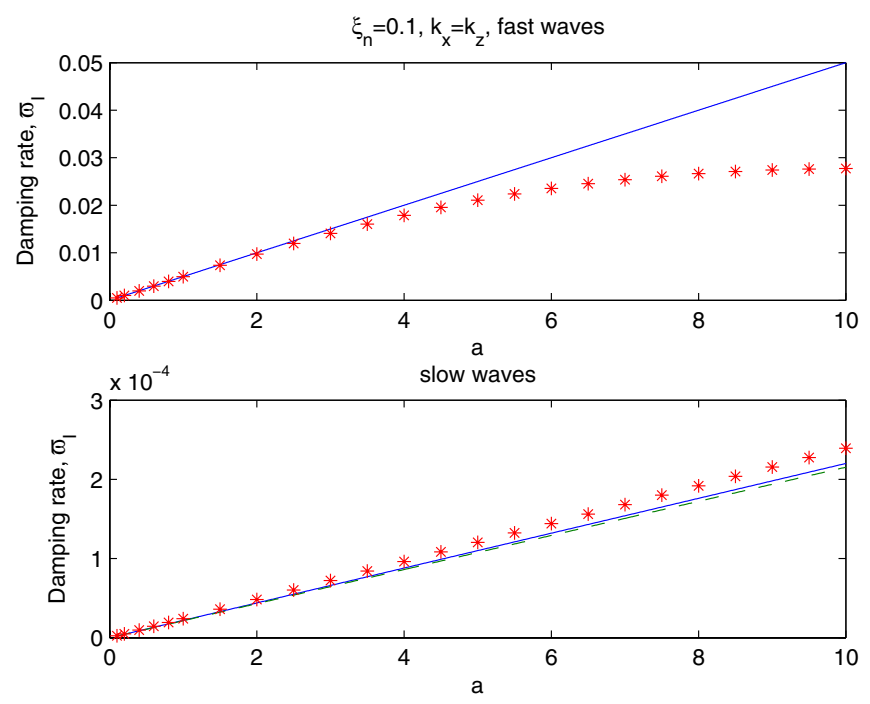

Fig. 4. Damping rate of the fast (upper panel) and slow (lower panel) magneto-acoustic waves, i.e. with the imaginary part of $\omega$ normalized by $k v_{\mathrm{A}}$, vs. the normalized wavenumber $a=k_{z} v_{\mathrm{A}} / v_{\text {in. }}$. The blue solid lines correspond to the solution of the single-fluid dispersion relation and the dashed line corresponds to the slow magneto-acoustic damping rate of Braginskii (the expressions used are from Forteza et al. 2007). Red asterisks are the solutions of the two-fluid dispersion relation, Eq. (57). The values are calculated for $10 \%$ of neutral hydrogen, $\xi_{\mathrm{n}}=0.1$, and for $c_{\mathrm{sn}} / v_{\mathrm{A}}=0.1$. The damping rates are calculated for waves propagating with a $45^{\circ}$ angle with regard to the magnetic field.

in Forteza et al. (2007). Our Figs. 4 and 5 show the damping rates of fast and slow magneto-acoustic waves vs. $a$ (i.e. $k$ ) for the propagation angles of $45^{\circ}$ and $0^{\circ}$, respectively. Red asterisks are the solutions of the two-fluid dispersion relation - Eq. (57). The blue solid lines correspond to the solutions of the singlefluid dispersion relation from Forteza et al. (2007). The dashed line corresponds to the slow magneto-acoustic damping rate of Braginskii (1965). Here we use $c_{\mathrm{sn}} / v_{\mathrm{A}}=0.1$ so the plasma $\beta$ is small enough. The neutral hydrogen concentration is taken to be $10 \%$. The fast magneto-acoustic waves have essentially the same dynamics as the Alfvén waves. For the smaller wave numbers (or lower frequencies) the two-fluid and single-fluid approaches give the same results, but for the larger wave numbers the damping rate is decreased in the two-fluid description as in the case of Alfvén waves. In contrast, the slow magneto-acoustic waves have similar damping rates in both approaches. There is a small discrepancy between the damping rates for the waves propagating with $45^{\circ}$ degree about the magnetic field, but all three cases (two-fluid waves, single-fluid waves, and energy consideration) yield similar results. The parallel propagation reveals an interesting result: the two-fluid solutions are exactly the same as those obtained by Braginskii (the damping rate obtained by Forteza et al. 2007 is zero for the parallel propagation). Therefore, the discrepancy between the damping rates of the slow magnetoacoustic waves obtained by Forteza et al. 2007) and Braginskii (1965) is again caused by neglecting the inertial terms in the momentum equation of relative velocity (Eq. (A.6)). Braginskii (1965) used the energy equation to calculate the damping rate, therefore his solution agrees with that obtained in our two-fluid approach.

Figure 6 shows the comparison of the damping rates in the two-fluid approach and those obtained by Braginskii (1965) for $\xi_{\mathrm{n}}=0.5, c_{\mathrm{sn}} / v_{\mathrm{A}}=0.1$ and parallel propagation. The fast magneto-acoustic waves have the same behavior as the Alfvén
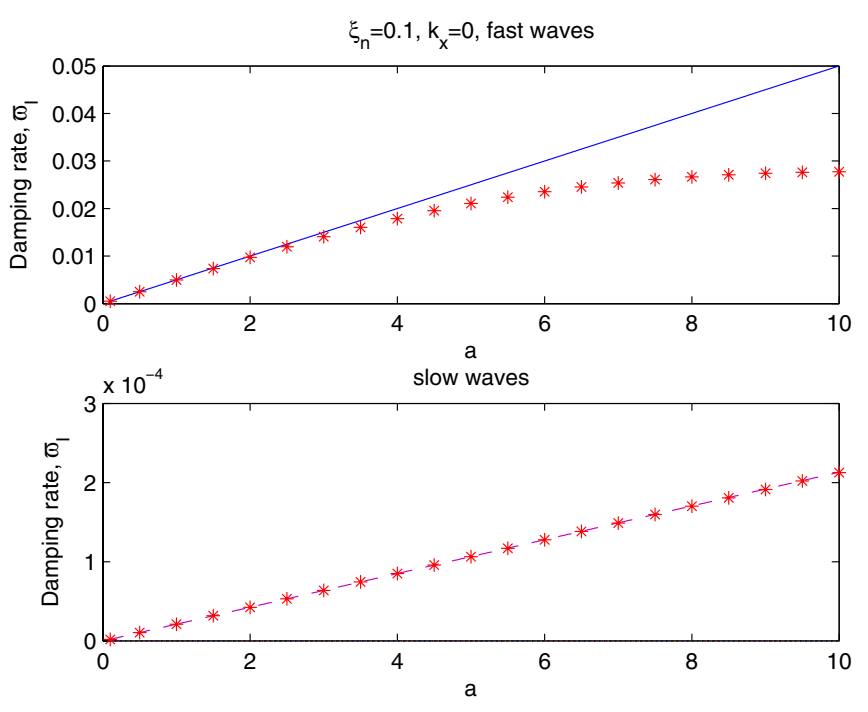

Fig. 5. The same as in Fig. 4 but for the parallel propagation, i.e. $k_{x}=0$.
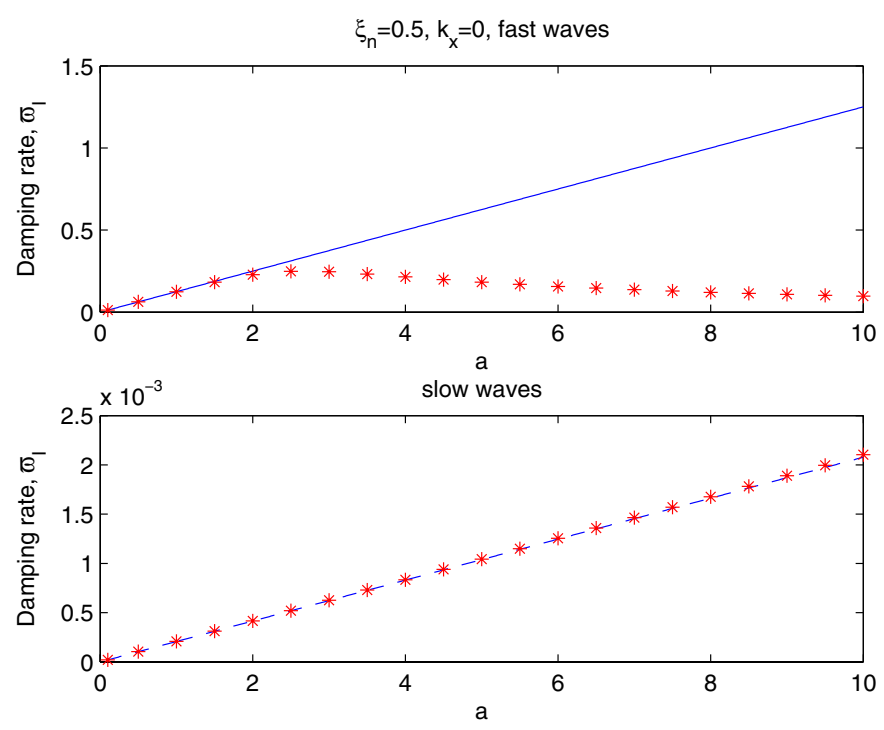

Fig. 6. Damping rate (the imaginary part of the normalized frequency $\left.\varpi=\omega / k_{z} v_{\mathrm{A}}\right)$ of fast (upper panel) and slow (lower panel) magnetoacoustic waves vs. the normalized wavenumber $a=k_{z} v_{\mathrm{A}} / v_{\text {in }}$. Red asterisks are the solutions of the two-fluid dispersion relation. Blue lines correspond to the solutions of Braginskii. The values are calculated for $50 \%$ of neutral hydrogen and $c_{\mathrm{sn}} / v_{\mathrm{A}}=0.1$. The damping rates are calculated for the waves that propagate along the magnetic field.

waves (see lower panel of Fig. 1), which is significantly different from Braginskii (1965) and Forteza et al. (2007). But the slow magneto-acoustic waves have the same damping rate as those of Braginskii (1965). On the other hand, the damping rate of slow magneto-acoustic waves becomes different from the solution of Braginskii (1965) for higher plasma $\beta$. Figure 7 shows the same as Fig. 6 , but for $c_{\mathrm{sn}} / v_{\mathrm{A}}=0.5$. The damping rate of the slow magneto-acoustic waves now begins to deviate from the solution of Braginskii for higher wave numbers. The behavior of fast magneto-acoustic waves remains the same.

\section{Discussion}

Some parts of the solar atmosphere contain a large number of neutral atoms: most of the atoms are neutral at the photospheric 
level, but the ionization degree rapidly increases with height owing to the increased temperature. Solar prominences also contain neutral atoms. Neutral atoms may change the dynamics of the plasma through collision with charged particles. For timescales longer than the ion-neutral collision time, the partially ionized plasma can be considered as one fluid, because collisions between neutrals and charged particles lead to the rapid coupling of the two fluids. Then the equation of motion is written for the center-of-mass velocity, and the motion of the species is considered as diffusion with a low velocity compared with the velocity of the center-of-mass. The corresponding collision terms appear in the equation of motion for the relative velocity (between ions and neutrals) and in the generalized Ohm's law. Neglecting the inertial term in the equation of motion for the relative velocity, one simplifies the equations, and a traditional induction equation with Cowling conductivity is obtained (Braginskii 1965; Khodachenko et al. 2004). The inertial terms (left-hand side terms in Eq. (A.6)) are smaller than the collision term (the last term in the same equation), but become comparable for time-scales near the ion-neutral collision time. Therefore, it can be neglected only for longer time-scales.

But for the time-scales of less than the ion-neutral collision time, both fluids may behave independently and the single-fluid approximation is not valid any more. Accordingly the two-fluid approximation, when ion-electron and neutral atom gases are treated as separate fluids, should be considered when one tries to model the processes in partially ionized plasmas.

The normal mode analysis of the two-fluid partially ionized plasma shows that frequencies and damping rates of lowfrequency MHD waves agree well with those found in the singlefluid approach. However, the waves with higher frequency than the ion-neutral collision frequency show a significantly different behavior. Alfvén and fast magneto-acoustic waves have maximal damping rates in a particular frequency interval, which peaks, for example, at the frequency $\omega=2.5 v_{\text {in }}$, ( $v_{\text {in }}$ is the ion-neutral collision frequency) for $\xi_{\mathrm{n}}=0.5$ and at the frequency $\omega=10 v_{\text {in }}$ for $\xi_{\mathrm{n}}=0.1$. The damping rates are reduced for the higher frequency part of wave spectrum (note that the damping rates are linearly increased in the single-fluid approach). Therefore, the statements concerning the damping of high-frequency Alfvén waves in the solar chromosphere through ion-neutral collisions should be revised. A careful analysis is needed to study the damping of high-frequency Alfvén waves for a realistic height profile of the ionization degree in the chromosphere.

Another important point concerning the Alfvén waves in partially ionized plasma is the cut-off wave-number, which appears in the single-fluid approach (Barcélo et al. 2010). Barcélo et al. (2010) found that the Alfvén waves with larger wave numbers than the cut-off value are evanescent in partially ionized and resistive plasmas. However, our two-fluid analysis shows that there is no cut-off wave number owing to ambipolar diffusion (see Fig.1, red asterisks). Therefore, the appearance of a cut-off wave number in the single-fluid approach is the result of neglecting of the inertial term in the equation of motion for the relative velocity. It is possible that the cut-off wave number that arises because of an usual magnetic resistivity is caused by neglecting the electron inertia, therefore the cut-off may completely disappear in a three-fluid approach. The cut-off wave numbers also appear for fast magneto-acoustic waves in partially ionized and resistive plasmas (Barcélo et al. 2010). We suggest that this may also be caused by neglecting the inertial term. However, this point needs further study.

The two-fluid approach reveals two different slow magnetoacoustic modes when the slow wave time-scale becomes shorter
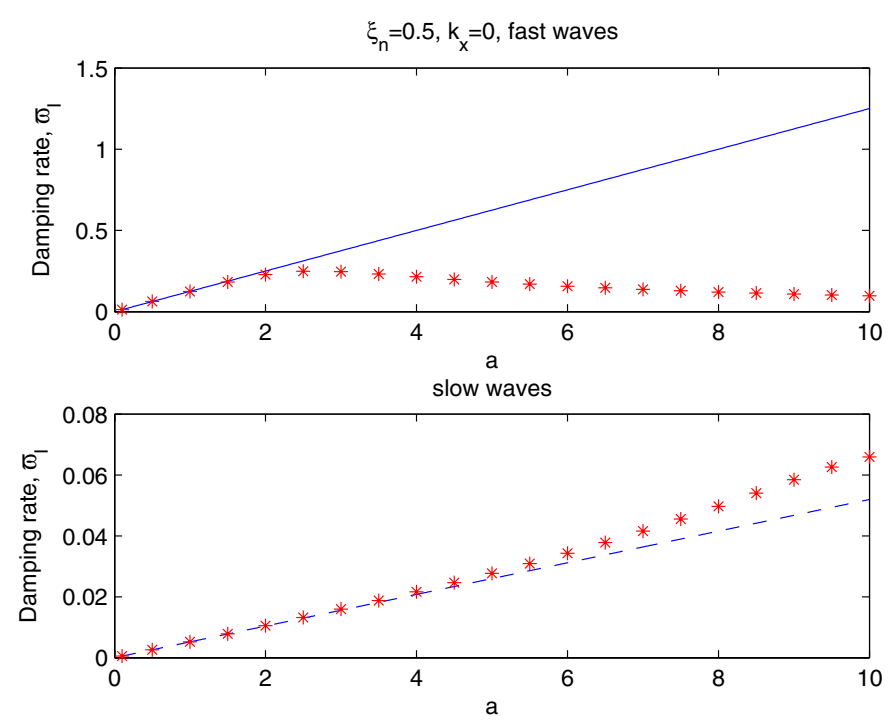

Fig. 7. The same as in Fig. 6, but for $c_{\mathrm{sn}} / v_{\mathrm{A}}=0.5$.

than the ion-neutral collision time (Fig. 3). The different slow modes correspond to ion-electron and neutral fluids. But only one slow magneto-acoustic mode remains at the lower frequency range as in the commonly used single-fluid approach. This is easy to understand physically. If the wave frequency is lower than the ion-neutral collision frequency, the two fluids are coupled through collisions and only one slow magneto-acoustic wave appears. The mode connected with the neutral fluid ("neutral" slow mode) has only an imaginary frequency in this range of the wave spectrum (not shown in Fig. 3). This means that any slow wave-type change (density, pressure) in the neutral fluid is damped faster than the wave period owing to collisions with ions. The "neutral" slow magneto-acoustic wave has similar properties as the ion magneto-acoustic waves.

The two-fluid approach of partially ionized plasma clarifies the uncertainty concerning the damping rate of slow magnetoacoustic waves found in the single-fluid approach. We found that the normal mode analysis and energy consideration method (used by Braginskii 1965) leads to different expressions for the damping rate of slow waves (Forteza et al. 2007). We found that the damping rate obtained in the two-fluid approach agrees well with the damping rate of Braginskii, which is derived from the energy treatment (see lower panels of Figs. 5-6). Therefore, it seems that the discrepancy is again caused by neglecting the inertial terms in the equation of motion for the relative velocity in the single-fluid approach. Braginskii (1965) used the general energy method for the estimation of damping rates, and this is probably the reason why his results agree with those found in the two-fluid approach.

Here we have considered only neutral hydrogen as a component of partially ionized plasma. However, other neutral atoms, for example neutral helium, may have important effects in the MHD wave damping processes. Soler et al. (2010) made the first attempt to include the neutral helium in the single-fluid description of prominence plasma. They concluded that the neutral helium has no significant influence on the damping of MHD waves. However, the two-fluid approach may give some more details about the effects of neutral helium on MHD waves, therefore it is important to study this point in the future. 


\section{Conclusions}

Frequencies and damping rates of low-frequency MHD waves in the two-fluid description are similar to those obtained in the single-fluid approach. But high-frequency waves (with a higher frequency than the ion-neutral collision frequency) have a completely different behavior.

Alfvén and fast magneto-acoustic waves have maximal damping rates at some frequency interval that peaks at a particular frequency. The peak frequency is $2.5 v_{\text {in }}$, where $v_{\text {in }}$ is the ion-neutral collision frequency, for $50 \%$ of neutral hydrogen. For $10 \%$ of neutral hydrogen, the peak frequency is shifted to $10 v_{\text {in }}$. The damping rate is reduced for higher frequencies, therefore the damping of high-frequency Alfvén waves in the solar chromosphere with a realistic height profile of the ionization degree needs to be revised in future.

There are two types of slow magneto-acoustic waves in the high-frequency part of the wave spectrum: one connected with the ion-electron fluid and another with the fluid of neutrals.

There is no cut-off frequency of Alfvén waves because of ambipolar diffusion. The cut-off frequency found in the singlefluid approach is caused by neglecting the inertial terms in the momentum equation of relative velocity.

The damping rate of slow magneto-acoustic waves is similar to Braginksii (1965) in low plasma $\beta$ approximation. The deviation from the Braginskii formula found by the normal mode analysis in the single-fluid approach (Forteza et al. 2007) is probably caused by neglecting the inertial terms.

Acknowledgements. The work was supported by the Austrian Fonds zur Förderung der wissenschaftlichen Forschung (project P21197-N16). T.V.Z. also acknowledges financial support from the Georgian National Science Foundation (under grant GNSF/ST09/4-310).

\section{Appendix A: Single-fluid equations}

We use the total velocity (i.e. velocity of center of mass)

$\boldsymbol{V}=\frac{\rho_{\mathrm{i}} \boldsymbol{V}_{\mathrm{i}}+\rho_{\mathrm{n}} \boldsymbol{V}_{\mathrm{n}}}{\rho_{\mathrm{i}}+\rho_{\mathrm{n}}}$

the relative velocity

$w=V_{\mathrm{i}}-V_{\mathrm{n}}$

and the total density

$\rho=\rho_{\mathrm{i}}+\rho_{\mathrm{n}}$.

Equation (20)-(25) and (27) lead to the system

$\frac{\partial \rho}{\partial t}+\nabla \cdot(\rho \boldsymbol{V})=0$

$\rho \frac{\partial \boldsymbol{V}}{\partial t}+\rho(\boldsymbol{V} \cdot \nabla) \boldsymbol{V}=-\nabla p+\frac{1}{c} \boldsymbol{j} \times \boldsymbol{B}-\nabla \cdot\left(\xi_{\mathrm{i}} \xi_{\mathrm{n}} \rho \boldsymbol{w} \boldsymbol{w}\right)$,

$$
\begin{aligned}
& \frac{\partial \boldsymbol{w}}{\partial t}+(\boldsymbol{V} \cdot \nabla) \boldsymbol{w}+(\boldsymbol{w} \cdot \nabla) \boldsymbol{V}+\xi_{\mathrm{n}}(\boldsymbol{w} \cdot \nabla) \boldsymbol{w}-(\boldsymbol{w} \cdot \nabla) \xi_{\mathrm{i}} \boldsymbol{w} \\
& =-\left(\frac{\nabla p_{\mathrm{ie}}}{\rho \xi_{\mathrm{i}}}-\frac{\nabla p_{\mathrm{n}}}{\rho \xi_{\mathrm{n}}}\right)+\frac{1}{c \rho \xi_{\mathrm{i}}} \boldsymbol{j} \times \boldsymbol{B}+\frac{\alpha_{\mathrm{en}}}{e n_{\mathrm{e}} \rho \xi_{\mathrm{i}} \xi_{\mathrm{n}}} \boldsymbol{j}-\frac{\alpha_{\mathrm{n}}}{\rho \xi_{\mathrm{i}} \xi_{\mathrm{n}}} \boldsymbol{w} \\
& \frac{\partial p}{\partial t}+(\boldsymbol{V} \cdot \nabla) p+\gamma p \nabla \cdot \boldsymbol{V}-\xi_{\mathrm{i}}(\boldsymbol{w} \cdot \nabla) p-\gamma p \nabla \cdot\left(\xi_{\mathrm{i}} \boldsymbol{w}\right) \\
& +(\boldsymbol{w} \cdot \nabla) p_{\mathrm{ie}}+\gamma p_{\mathrm{ie}} \nabla \cdot \boldsymbol{w}=(\gamma-1) \frac{\alpha_{\mathrm{ei}}+\alpha_{\mathrm{en}}}{e^{2} n_{\mathrm{e}}^{2}} j^{2}+(\gamma-1) \alpha_{\mathrm{n}} w^{2} \\
& -(\gamma-1) \frac{2 \alpha_{\mathrm{en}}}{e n_{\mathrm{e}}} \boldsymbol{j} \boldsymbol{w}+\frac{1}{e n_{\mathrm{e}}}(\boldsymbol{j} \cdot \nabla) p_{\mathrm{e}}+\gamma p_{\mathrm{e}} \nabla \cdot \frac{j}{e n_{\mathrm{e}}} \\
& -(\gamma-1) \nabla \cdot\left(\boldsymbol{q}_{\mathrm{i}}+\boldsymbol{q}_{\mathrm{e}}+\boldsymbol{q}_{\mathrm{n}}\right), \\
& \frac{\partial \boldsymbol{B}}{\partial t}=\nabla \times(\boldsymbol{V} \times \boldsymbol{B})+\nabla \times\left(\frac{c \nabla p_{\mathrm{e}}}{e n_{\mathrm{e}}}\right)-\nabla \times(\eta \nabla \times \boldsymbol{B}) \\
& -\nabla \times\left(\frac{\boldsymbol{j} \times \boldsymbol{B}}{e n_{\mathrm{e}}}\right)+\nabla \times\left(\frac{c \alpha_{\mathrm{en}} \boldsymbol{w}}{e n_{\mathrm{e}}}\right)+\nabla \times\left(\xi_{\mathrm{n}} \boldsymbol{w} \times \boldsymbol{B}\right)
\end{aligned}
$$

where $p=p_{\mathrm{e}}+p_{\mathrm{i}}+p_{\mathrm{n}}, \xi_{\mathrm{i}}=\rho_{\mathrm{i}} / \rho, \xi_{\mathrm{n}}=\rho_{\mathrm{n}} / \rho$ and $\alpha_{\mathrm{n}}=\alpha_{\text {in }}+\alpha_{\text {en }}$. Ohm's law is now

$\boldsymbol{E}+\frac{1}{c} \boldsymbol{V} \times \boldsymbol{B}+\frac{1}{e n_{\mathrm{e}}} \nabla p_{\mathrm{e}}=\frac{\alpha_{\mathrm{ei}}+\alpha_{\mathrm{en}}}{e^{2} n_{\mathrm{e}}^{2}} \boldsymbol{j}-\frac{\alpha_{\mathrm{en}}}{e n_{\mathrm{e}}} \boldsymbol{w}+\frac{1}{c e n_{\mathrm{e}}} \boldsymbol{j} \times \boldsymbol{B}$

$-\frac{\xi_{\mathrm{n}}}{c} \boldsymbol{w} \times \boldsymbol{B}$

Neglecting the inertia terms, i.e. all left hand side terms in Eq. (A.6), we have

$$
\boldsymbol{w}=-\frac{\boldsymbol{G}}{\alpha_{\mathrm{n}}}+\frac{\xi_{\mathrm{n}}}{c \alpha_{\mathrm{n}}} \boldsymbol{j} \times \boldsymbol{B}+\frac{\alpha_{\mathrm{en}}}{e n_{\mathrm{e}} \alpha_{\mathrm{n}}} \boldsymbol{j}
$$

Then the induction equation takes the form

$$
\begin{aligned}
\frac{\partial \boldsymbol{B}}{\partial t}= & \nabla \times(\boldsymbol{V} \times \boldsymbol{B})+\frac{c}{e} \nabla \times\left(\frac{\nabla p_{\mathrm{e}}-\epsilon \boldsymbol{G}}{n_{\mathrm{e}}}\right)-\nabla \times\left(\eta_{T} \nabla \times \boldsymbol{B}\right) \\
& -\frac{c}{4 \pi e} \nabla \times\left(\frac{1-2 \epsilon \xi_{\mathrm{n}}}{n_{\mathrm{e}}}(\nabla \times \boldsymbol{B}) \times \boldsymbol{B}\right)-\nabla \times\left(\frac{\xi_{\mathrm{n}}}{\alpha_{\mathrm{n}}} \boldsymbol{G} \times \boldsymbol{B}\right) \\
& +\nabla \times\left(\frac{\xi_{\mathrm{n}}^{2}}{4 \pi \alpha_{\mathrm{n}}}((\nabla \times \boldsymbol{B}) \times \boldsymbol{B}) \times \boldsymbol{B}\right),
\end{aligned}
$$

where $\epsilon=\alpha_{\mathrm{en}} / \alpha_{\mathrm{n}}, \boldsymbol{G}=\xi_{\mathrm{n}} \nabla p_{\mathrm{ei}}-\xi_{\mathrm{i}} \nabla p_{\mathrm{n}}$ and

$\eta_{T}=\frac{c^{2}}{4 \pi \sigma}=\frac{c^{2}}{4 \pi e^{2} n_{\mathrm{e}}^{2}}\left(\alpha_{\mathrm{ei}}+\alpha_{\mathrm{en}}-\frac{\alpha_{\mathrm{en}}^{2}}{\alpha_{\mathrm{n}}}\right)$.

These equations are traditionally used for the description of partially ionized plasmas.

\section{References}

Arber, T. D., Haynes, M., \& Leake, J. E. 2007, ApJ, 666, 541 Barcélo, S., Carbonell, M., \& Ballester, J. L. 2010, A\&A, 525, A60 Braginskii, S. I. 1965, Rev. Plasma Phys., 1, 205 Carbonell, M., Forteza, P., Oliver, R., \& Ballester, J. L. 2010, A\&A, 515, A80 De Pontieu, B., \& Haerendel, G. 1998, A\&A, 338, 729

Fontenla, J. M., Avrett, E. H., \& Loeser, R. 1990, ApJ, 355, 700 
T. V. Zaqarashvili et al.: MHD waves in partially ionized plasma

Forteza, P., Oliver, R., Ballester, J. L., \& Khodachenko, M. L. 2007, A\&A, 461, 731

Forteza, P., Oliver, R., \& Ballester, J. L. 2008, A\&A, 492, 223

Goedbloed, H., \& Poedts, S. 2004, Principles of Magnetohydrodynamics (Cambridge University Press)

Khodachenko M. L., \& Rucker, H. O. 2005, Adv. Space Res., 36, 1561

Khodachenko M. L., \& Zaitsev, V. V. 2002, Astrophys. Space Sci., 279, 389

Khodachenko M. L., Arber, T. D., Rucker, H. O., \& Hanslmeier, A. 2004, A\&A, 422,1073
Haerendel G. 1992, Nature, 360, 241

James, S. P., \& Erdélyi, R. 2002, A\&A, 393, L11

James, S. P., Erdélyi, R., \& De Pontieu, B. 2004, A\&A, 406, 715

Leake, J. E., \& Arber, T. D. 2006, A\&A, 450, 805

Leake, J. E., Arber, T. D., \& Khodachenko, M. L. 2005, A\&A, 442, 1091

Soler, R., Oliver, R., \& Ballester, J. L. 2009a, ApJ, 699, 1553

Soler, R., Oliver, R., \& Ballester, J. L. 2009b, ApJ, 707, 662

Soler, R., Oliver, R., \& Ballester, J. L. 2010, A\&A, 512, A28

Vranjes, J., Poedts, S., Pandey, B. P., \& de Pontieu, B. 2008, A\&A, 478, 553 